\title{
Solid State Transitions in Alkali Alkanoates: Diffractometric and Conductometric Measurements on Lithium, Sodium and Potassium Propanoates
}

\author{
A. Cingolani, G. Spinolo, and M. Sanesi \\ Centro di Studio per la Termodinamica ed Elettrochimica dei Sistemi Salini Fusi \\ e Solidi del C.N.R. c/o Istituto di Chimica Fisica e di Elettrochimica \\ dell'Università, Pavia, Italy
}

Z. Naturforsch. 34 a, 575-578 (1979); received March 26, 1979

\begin{abstract}
Conductometric measurements on solid lithium, sodium and potassium propanoates and $\mathrm{X}$-ray powder diffraction measurements on the sodium and potassium salts have been performed over temperature ranges including solid state transitions. The temperature dependence of the transport and lattice parameters of the different crystalline phases and the changes of the same parameters in correspondence with phase transitions have been put into evidence.
\end{abstract}

\section{Introduction}

The melting of the alkali salts of the alkane carboxylic acids is in several cases a stepwise process, involving intermediate stages between the true crystalline solid and the isotropic liquid; in particular the formation and properties of mesomorphic phases have been studied from several points of view and with different techniques (X-ray, dilatometry, electrical conductance, viscosity, etc.; see, e.g. [1], [2]).

Several crystalline forms are often encountered with these salts; for what concerns the structural features of the different polymorphs, a number of investigations on salts with long chain anions may be found in the literature [3]. In order to better understand the role played by the ionic portion of the molecule, the lower homologues ought to be considered preferably and, among these, the propanoates which have, unlike the acetates, possibilities of configurational motions that markedly affect the thermal behaviour.

It has been pointed out [4] that investigations concerning solid state transitions (sstr) are of remarkable interest for understanding the mechanism of entropy increase in this salt family. Such sstr's, however, are often characterized by very little structural changes (to which small heat effects correspond): in these cases investigations of equilibrium properties alone might not suffice and be paralleled by measurements of some transport property.

Reprint requests to Prof. M. Sanesi, Istituto di Chimica Fisica e di Elettrochimica dell' Università, Viale Taramelli, 16, I-27100 Pavia (Italy).

$0340-4811 / 79 / 0500-0575 \$ 01.00 / 0$
The present paper deals with diffractometric and conductometric measurements on lithium, sodium and potassium propanoates (which have been proved not to exist as anisotropic liquids [5]), aiming at putting into evidence the changes of lattice and transport parameters connected to sstr's.

\section{Experimental}

The salts investigated were prepared and handled according to [5].

The lattice parameters were determined by polythermal X-ray powder diffractometry, employing a Philips PW 1011 Diffractometer (with Cu fine focus tube, usually operating at $38 \mathrm{kV}$ and $18 \mathrm{~mA}$; proportional detector; ratemeter; timerscaler; and recorder) and a polythermal chamber built up in this Institute and described elsewhere [6].

Powder patterns were indexed following De Wolff's method [7]: the calculated densities were in accordance with the pycnometric ones $(d=$ $1.49 \pm 0.03$ and $1.45 \pm 0.01 \mathrm{~g} \mathrm{~cm}^{-3}$ for the $\mathrm{Na}$ and $\mathrm{K}$ salt, respectively). The reliability index [8] of the unit cells was seldom between 15 and 25 , but usually larger than the latter number. The lattice parameters were obtained by means of a computer program which minimizes the weighted sum of the squares of the differences between experimental and calculated $Q$ values $\left(Q=4 \sin ^{2} \theta / \lambda^{2}\right)$.

The measurements of conductance, $G$, were carried out with a Wayne-Kerr Autobalance Precision Bridge mod. B $331 \mathrm{Mk}$ II (frequency: $1592 \mathrm{~Hz}$; conductance range: $10^{-10}-10^{-2} \mathrm{~S}$ ) on pellet-shaped cells $(8 \mathrm{~mm}$ in diameter; $5-7 \mathrm{~mm}$ thick) obtained by pressing the salt powder between 
two silver powder layers. Details on the cells and on the cell-furnace assembly have been reported previously [9]. Heating was accomplished by means of an $\mathrm{L} \& \mathrm{~N}$ temperature programmer and power unit. The outputs from the measuring thermocouple (type $\mathrm{K}$ ) and from the bridge were fed to the same recorder to detect continuously (heating rate: $\sim 2 \mathrm{~K} \mathrm{~min}^{-1}$ ) the temperature dependence of the solid phase conductance. As a rule, different experimental runs were carried out on four or more pellets for each salt.

Derivative plots ( $\mathrm{d} \log G / \mathrm{d} T$ vs. $T$ ) were employed for a better characterization of the sstr regions, whereas a linearized least-squares analysis of the Arrhenius plots $(\log G$ vs. $1 / T)$ allowed to evaluate the activation energies, $E_{\mathrm{a}}$, of the conductance process. To a first approximation the latter might be considered as equivalent to the activation energies for specific conductivity, $E_{\varkappa} *$.

An approximate knowledge of the cell constants (from the geometrical dimensions of the pellets) allowed to obtain the order of magnitude of the specific conductivity, $x$.

\section{Results and Discussion}

The sstr temperatures of the salts here concerned, as detected by differential scanning calorimetry (DSC) between $110 \mathrm{~K}$ and the melt region [5], were as follows:

Li propanoate $\left(T_{\mathrm{F}}=606.8 \mathrm{~K}\right): T_{\mathrm{A}}=553 \pm 2 \mathrm{~K}$, Na propanoate $\left(T_{\mathrm{F}}=562.4 \mathrm{~K}\right): T_{\mathrm{A}}=494 \pm 1 \mathrm{~K}$;

$\mathrm{K}$ propanoate $\left(T_{\mathrm{F}}=638.3 \mathrm{~K}\right)$ :

$$
T_{\mathrm{B}}=470.2 \pm 0.6 \mathrm{~K} \text {, }
$$

$$
\begin{aligned}
& T_{\mathrm{A}}=352.5 \pm 0.6 \mathrm{~K} ; \\
& T_{\mathrm{B}}=258 \pm 2 \mathrm{~K} .
\end{aligned}
$$

The several polymorphs of each salt are indicated hereafter as phase I, phase II, etc., starting from that stable at higher temperature.

The conductance measurements were carried out in the temperature ranges $480-540$ for $\mathrm{Li}$ propanoate, $420-540$ for $\mathrm{Na}$ propanoate and $320-630 \mathrm{~K}$ for $\mathrm{K}$ propanoate, the lowest limit being imposed by the instrumental sensitivity. It was not possible, therefore, to perform measurements on phase III of $\mathrm{K}$ propanoate, neither to detect for the same salt sstr $\mathrm{A}$, in correspondence

* This can be inferred from the expansion coefficients of the solid salts, as deduced from the temperature dependence of the lattice parameters. to which the change in the conductance values was below the experimental accuracy.

The derivative plots $\mathrm{d} \log G / \mathrm{d} T$ vs. $T$ are shown in Figure 1: good agreement with the DSC sstr temperatures is apparent. The main results are summarized in Table 1, where the specific conductivity values of the phases in equilibrium at the

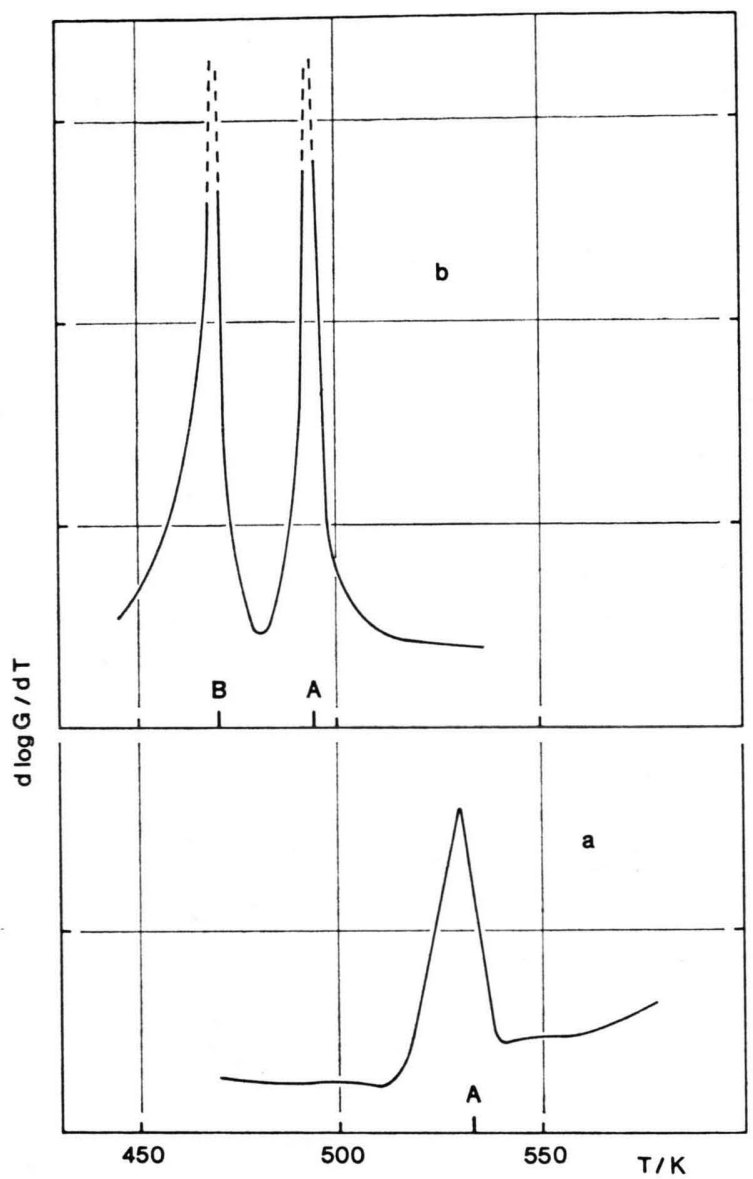

Fig. 1. Conductometric detection of the sstr temperatures in $\mathrm{Li}$ propanoate (a) and $\mathrm{Na}$ propanoate (b). The DSC values are indicated by vertical lines on the abscissa.

Table 1. Electrical conductance parameters for $\mathrm{Li}$ and $\mathrm{Na}$ propanoate solid phases.

\begin{tabular}{lrllr}
\hline Salt & Phase & $\begin{array}{l}\text { Tempera- } \\
\text { ture range } \\
{[\mathrm{K}]}\end{array}$ & $\frac{\varkappa_{\mathrm{tr}}}{\mathrm{S} \mathrm{m} \mathrm{m}^{-1}}$ & $\frac{E_{\varkappa}}{\mathrm{kJ} \mathrm{mol}^{-1}}-$ \\
\hline $\begin{array}{l}\text { Li pro- } \\
\text { panoate }\end{array}$ & II & $530-580$ & $3.6 \cdot 10^{-6}$ & $149 \pm 8$ \\
Na pro- & I & $480-530$ & $6.5 \cdot 10^{-7}$ & $61 \pm 8$ \\
panoate & II & $470-494$ & $1.3 \cdot 10^{-4}$ & $123 \pm 8$ \\
\hline
\end{tabular}


transition temperature, $\varkappa_{\text {tr }}$, are reported along with the activation energies of the same phases. It was not possible to evaluate $E_{\varkappa}$ for phase III of $\mathrm{Na}$ propanoate and phase I of $\mathrm{K}$ propanoate, since $\log G$ is not a linear function of $1 / T$ in these cases. For the latter phase the measured $\varkappa$ values ranged from $\sim 1.7 \cdot 10^{-8} \mathrm{~S} \mathrm{~m}^{-1}$ at $373 \mathrm{~K}$ to $\sim 2 \cdot 10^{-5} \mathrm{~S} \mathrm{~m}^{-1}$ at $573 \mathrm{~K}$. The present $E_{\varkappa}$ value for phase I of $\mathrm{Na}$ propanoate is in satisfactory agreement with that reported by Duruz et al. [2] $\left(112 \mathrm{~kJ} \mathrm{~mol}^{-1}\right)$ for a narrow temperature range $(542-558 \mathrm{~K})$ close to the melting point.

It is to be remarked that the activation energy in Li propanoate impressively increases in the transition from the lower to the higher temperature phase, whereas in the $\mathrm{Na}$ salt practically the same $E_{\varkappa}$ value is exhibited both by the I and II phase. The latter fact might be also related to the close similarity in the structure of these phases, as shown by the subsequent $\mathrm{X}$-ray measurements.
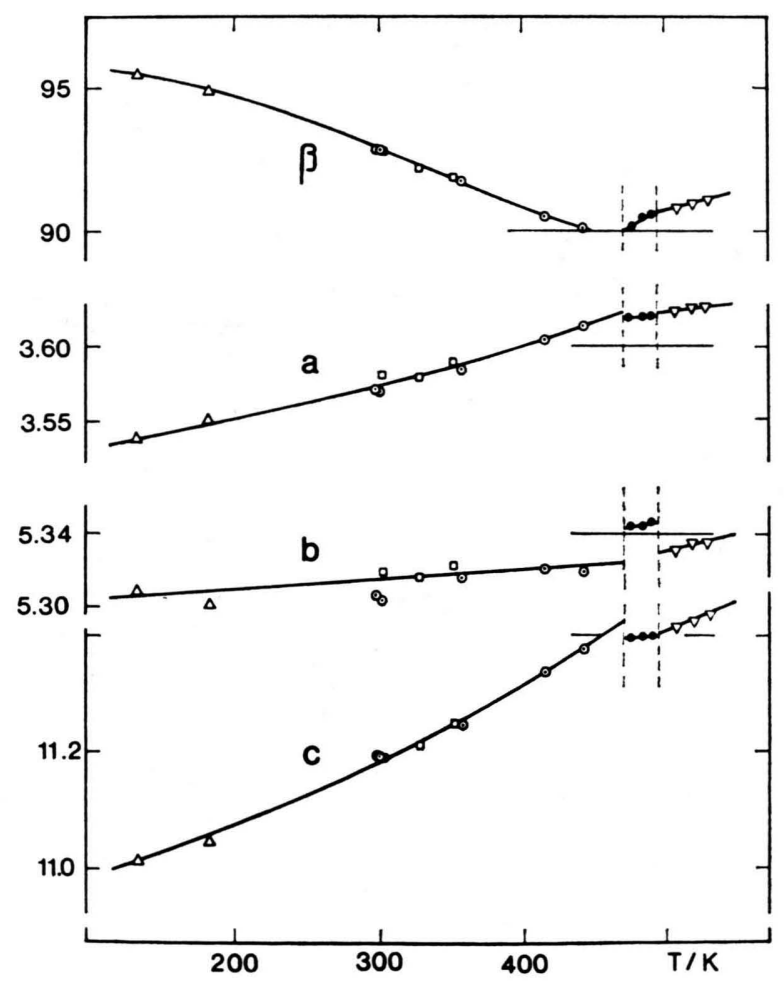

Fig. 2. Temperature dependence of the lattice parameters in phases I, II and III of $\mathrm{Na}$ propanoate ( $\mathrm{a}, \mathrm{b}$ and $\mathrm{c}$ in $\AA$, $\beta$ in degrees; different symbols refer to different investigated samples).
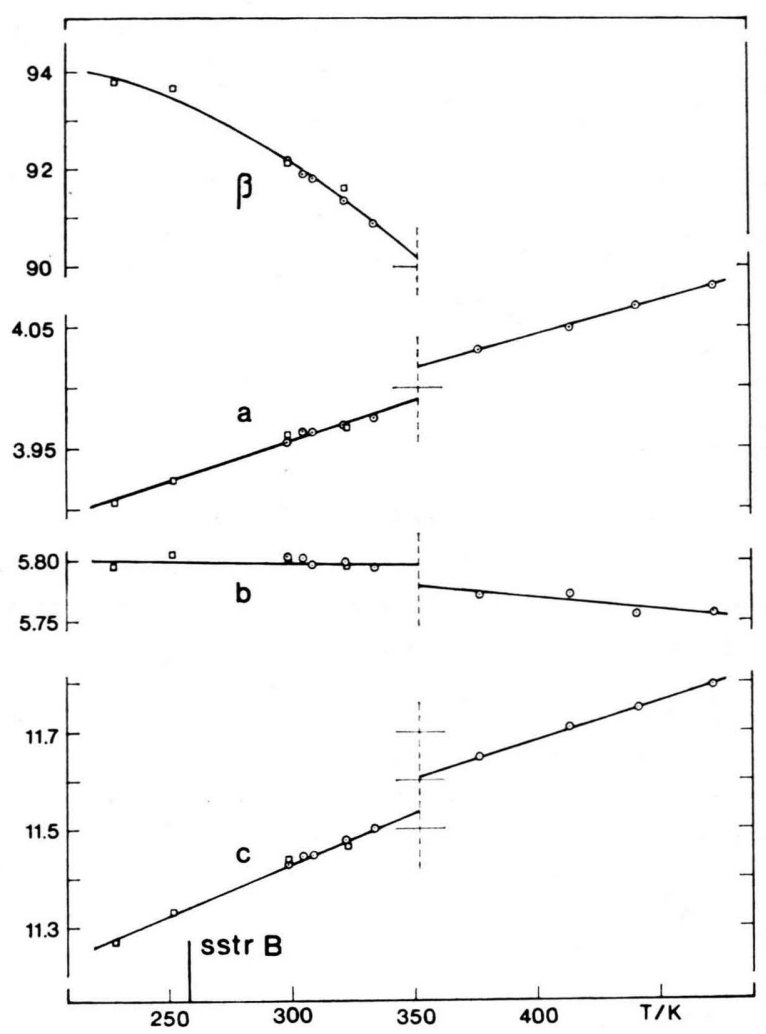

Fig. 3. Temperature dependence of the lattice parameters in phases I and II of $\mathrm{K}$ propanoate (a,b and $\mathrm{c}$ in $\AA, \beta$ in degrees; different symbols refer to different investigated samples). The points at temperatures below sstr B (indicated by a vertical line on the abscissa) were obtained from undercooled phase II samples.

The diffractometric investigation was performed in the temperature ranges $130-540$ and $130-470 \mathrm{~K}$ for the $\mathrm{Na}$ and the $\mathrm{K}$ salt, respectively.

With the exception of the low temperature phase III of $\mathrm{K}$ propanoate, for which indexing was not successful in spite of several trials, all powder diffraction patterns were similar and led to monoclinic (orthorhombic in phase $\mathrm{I}$ of $\mathbf{K}$ propanoate) unit cells containing two molecules.

The temperature dependence of the lattice constants is shown in Figs. 2 and 3 ; in Table 2 smoothed values at a few fixed temperatures are reported.

The X-ray results for phase III of Na propanoate agree with previous dilatometric measurements [2], whereas differences exist for what concerns the molar volume changes in correspondence to sstr's A and B. No satisfactory explanation of such dis- 
Table 2. Selected values of the lattice parameters for $\mathrm{Na}$ and $\mathrm{K}$ propanoate solid phases.

\begin{tabular}{lcllllr}
\hline Salt & \multirow{2}{*}{ Phase } & $\begin{array}{l}T \\
{[\mathrm{~K}]}\end{array}$ & $\begin{array}{l}a \\
{[\AA]}\end{array}$ & $\begin{array}{l}b \\
{[\AA]}\end{array}$ & $\begin{array}{l}c \\
{[\AA]}\end{array}$ & \multicolumn{1}{l}{$\begin{array}{l}\beta \\
{\left[{ }^{\circ}\right]}\end{array}$} \\
\hline Na pro- & III & 200 & 3.549 & 5.310 & 11.075 & 94.61 \\
panoate & & 300 & 3.573 & 5.317 & 11.184 & 92.82 \\
& & 400 & 3.600 & 5.324 & 11.313 & 90.82 \\
& & 470 & 3.623 & 5.328 & 11.416 & $(\cong 90)$ \\
& II & 480 & 3.621 & 5.357 & 11.397 & 90.30 \\
& I & 500 & 3.623 & 5.340 & 11.403 & 90.75 \\
K pro- & II & 273 & 3.939 & 5.799 & 11.369 & 93.02 \\
panoate & & 300 & 3.957 & 5.798 & 11.426 & 92.22 \\
& & 350 & 3.991 & 5.797 & 11.531 & 90.28 \\
& I & 355 & 4.020 & 5.776 & 11.613 & \\
& & 400 & 4.045 & 5.768 & 11.681 & \\
& & 450 & 4.073 & 5.758 & 11.756 & \\
\hline
\end{tabular}

crepancies was found, in spite of great experimental care. The possibility that the present data refer to metastable phases is to be discarded, since no changes in the patterns were observed after having kept the samples in the pertinent temperature range for reasonably long periods (up to a whole day): moreover, rather fast kinetics for the phase transitions III $\rightleftarrows$ II and II $\rightleftarrows$ I in Na propanoate had been revealed by DSC analysis [5].

[1] J. J. Duruz and A. R. Ubbelohde, Proc. Roy. Soc. London $330 \mathrm{~A}, 1$ (1972).

[2] J. J. Duruz, H. J. Michels, and A. R. Ubbelohde, Proc. Roy. Soc. London 322 A, 281 (1971).

[3] B. Gallot and A. Skoulios, Koll.-Z. Polym. 209, 164 (1966); 210, 143 (1966); 213, 143 (1966); 222, 51 (1968).

[4] A. R. Ubbelohde, Melting and Crystal Structure, Clarendon Press, Oxford 1965.

[5] P. Ferloni, M. Sanesi, and P. Franzosini, Z. Naturforsch. 30 a, 1447 (1975).
It is still to be noted that phases I and II of Na propanoate, besides exhibiting very close values of the cell parameters, gave markedly similar diffraction patterns (just slightly differing in the intensity ratios of the reflections).

The single-crystal structure determinations by Lomer et al. [10] on a few $\mathrm{K}$ salts indicate bidimensional close packings of carboxylate and alkali ions separated by layers of hydrocarbon chains. On the other hand, the investigations by Gallot and Skoulios [3] on the longer chain homologues put into evidence the influence of the conformational disorder on the long range packing. The cell dimensions determined in the present work are in agreement with the above authors' results: in particular the crystal structure of the phases considered here is of the same type as that termed "lamellaire crystalline" by Gallot and Skoulios. The absence of plastic or mesomorphic phases is clearly a consequence of the lower number of conformational degrees of freedom, while the changes in the lattice constants $a$ and $b$ (which define the dimensions of the ionic double layer) on passing from the $\mathrm{Na}$ to the $\mathrm{K}$ salt can satisfactorily be accounted for with the difference of the cationic radii.

[6] G. Spinolo, V. Massarotti, and G. Campari, to be published.

[7] P. M. De Wolff, Acta Cryst. 10, 590 (1957).

[8] O. Kennard, J. D. Hanawalt, A. J. C. Wilson, P. M. De Wolff, and V.A. Frank-Kamenetsky, J. Appl. Cryst. 4, 81 (1971).

[9] A. Schiraldi and G. Chiodelli, J. Phys. E: Sci. Instrum. 10, 596 (1977).

[10] T. R. Lomer, Acta Cryst. 5, 11 (1952); J. H. Dumbleton and T. R. Lomer, ibid. 19, 301 (1965). 